\title{
Frequency and voltage dependent electrical responses of poly(triarylamine) thin film-based organic Schottky diode
}

\author{
Khairul Anuar Mohamad ${ }^{1, *}$, Hang Tak Hoh $^{1}$, Afishah Alias ${ }^{1}$, Bablu Kumar Ghosh ${ }^{1}$, and Hisashi Fukuda ${ }^{2}$ \\ ${ }^{1}$ Faculty of Engineering, Universiti Malaysia Sabah, Jalan UMS, Kota Kinabalu, Sabah, 88400 Malaysia \\ ${ }^{2}$ Faculty of Engineering, Muroran Institute of Technology, 27-1 Mizumoto, Muroran, Hokkaido, 050-8585 Japan
}

\begin{abstract}
A metal-organic-metal (MOM) type Schottky diode based on poly (triarylamine) (PTAA) thin films has been fabricated by using the spin coating method. Investigation of the frequency dependent conductance-voltage $(G-V-f)$ and capacitance-voltage $(C-V-f)$ characteristics of the ITO/PTAA/Al MOM type diode were carried out in the frequency range from $12 \mathrm{~Hz}$ to $100 \mathrm{kHz}$ using an LCR meter at room temperature. The frequency and bias voltage dependent electrical response were determined by admittancebased measured method in terms of an equivalent circuit model of the parallel combination of resistance and capacitance $(R C$ circuit). Investigation revealed that the conductance is frequency and a bias voltage dependent in which conductance continuous increase as the increasing frequency, respectively. Meanwhile, the capacitance is dependent on frequency up to a certain value of frequency $(100 \mathrm{~Hz})$ but decreases at high frequency $(1-10 \mathrm{kHz})$. The interface state density in the Schottky diode was determined from $G-V$ and $C-V$ characteristics. The interface state density has values almost constant of $2.8 \times 10^{12} \mathrm{eV}^{-1} \mathrm{~cm}^{-2}$ with slightly decrease by increasing frequencies. Consequently, both series resistance and interface trap density were found to decrease with increasing frequency. The frequency dependence of the electrical responses is attributed the distribution density of interface states that could follow the alternating current (AC) signal.
\end{abstract}

\section{Introduction}

Organic semiconductor raises intense attention in producing thin film application devices like Schottky diodes, field-effect transistors, and solar cells, as a potential candidate for future emerging electronics analogous to conventional technology $[1,2]$. Organic semiconductor materials have advantages such as an easy fabrication process, low cost, mechanical flexibility, large area electronic applications, low temperature process and more versatile than inorganic semiconductor, which allowed a manipulation and the molecular level and building of eco-friendly and sustainable future electronics ranging from flexible and wearable electronics to energy-harvesting devices. Recently, solution-processed organic semiconductor, especially polymer, gained significant attention as new functional organic materials applied in the development and advancement in high performance and stability with reasonable electrical properties to meet the challenge due to their unique electronic and optical properties [3-5]. Among the family of polymer, poly(triarylamine) (PTAA) is an amorphous p-type polymer semiconductor, which offers a lot of advantages for device technologies such as low-cost materials, simple fabrication techniques and enables thin films at room temperature [6,7], but have not yet demonstrated the high performance device with excellence electrical properties such as mobility $[8,9]$, but is known as polymer binder that enable to form uniform polymer thin films in large areas [10,11]. Thus, metal/organic Schottky diodes are useful ways to investigate the influence of conduction mechanism to the electrical properties.

Despite the investigation of the organic-based devices in DC measurements, very little is known about their characteristics in $\mathrm{AC}$ operation on conduction or transport mechanism, charge carrier injection, relaxation process, disordered molecule and interfacial layers between molecules. Organic semiconductors showed strongly frequency-dependent electrical response in a wide frequency range, especially at low and intermediate frequencies due to the interface states or traps and surface polarization [12-15]. The formation of an interfacial layer between metal and organic semiconductor, a variation of the semiconductor surface and series resistance between contact interface cause deviations of the ideal diode behavior [16]. Therefore, parameters such as interface states and series resistance play an important role in the determination of reasonable electrical characteristics of the metal-organic-metal (MOM) type diode. The information of such parameters can be obtained from the conductance and capacitance methods such as conductance-voltage-frequency $(G-V-f)$ and capacitance-voltage-frequency $(C-V-f)$ measurements [17-20]. These measurements are common tools to characterize the quality of the insulator/organic interfaces and various measurement techniques are available for determining the interface trap density [2125]. The interface trap density and series resistance usually depend on frequency and cause frequency dispersion [17,26-29].

\footnotetext{
* Corresponding author: khairul@ums.edu.my
} 
In this work, an investigation of the frequency dependent electrical responses of ITO/PTAA/Al Schottky diode using the conductance-frequency $(G-f)$, the conductance-voltage $(G-V)$, the capacitancefrequency $(C-f)$ and the capacitance-voltage $(C-V)$ measurements are presented to elucidate information on the electrical parameters of the organic Schottky diode. The characterization and analysis of the ac response will give important information for the conduction process of the thin films.

\section{Experiments}

PTAA (Lumtec) without further purification, as shown in Figure 1, was dispersed in chloroform to generate 0.1 $\mathrm{wt} \%$ polymer solution. PTAA was soaked overnight until all solid materials were dissolved at a room temperature. Prior to thin film deposition, the glass as a substrate was soaked overnight in Decon90 and ultrasonically cleaned with ethanol, acetone and distilled water followed by drying with nitrogen gas. Then, a PTAA thin film was deposited by spin-coating at 500 $\mathrm{rpm}$ for $30 \mathrm{~s}$, and followed further sintering at $2000 \mathrm{rpm}$ for $50 \mathrm{~s}$ from a syringe with a filter to form an approximately 100-nm-thick thin film. Aluminum (Al) metal with a thickness of $150 \mathrm{~nm}$ was deposited on the spin-coated thin films through a designated mask in a vacuum chamber for top contact using sputtering technique. The area dimension of the diode was $0.5 \times 0.5$ $\mathrm{cm}^{2}$. The schematic diagram of the fabricated MOM type Schottky diode structure is shown in Figure 1. The frequency dependent conductance $(G-f)$ and capacitance $(C-f)$ were measured over a frequency range of $12 \mathrm{~Hz}-$ $100 \mathrm{kHz}$ using a precision LCR meter (GW Instek LCR821). The voltage dependent conductance $(G-V)$ and capacitance $(C-V)$ were carried out at the various bias voltages from $0.2-5.0 \mathrm{~V}$ with an $\mathrm{AC}$ oscillating wave. All measurements were recorded at the room temperature in an ambient condition.

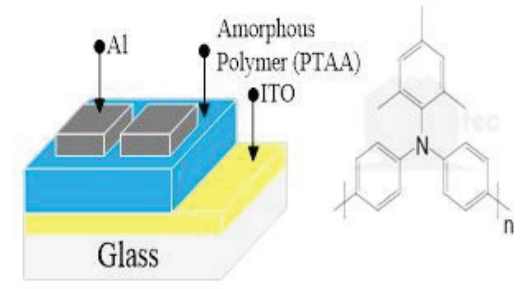

Fig. 1. Molecular structures of PTAA and schematic diagram of the ITO/PTAA/Al metal-organic-metal (MOM) type Schottky diode structure.

\section{Results and discussion}

\subsection{Frequency dependent conductance and capacitance}

The frequency dependent conductance and capacitance characteristics provide important information on the conduction process and these characteristics were obtained based on the following complex admittance equation in terms of an equivalent circuit model of the parallel combination of resistance and capacitance $(R C$ circuit) [24,25]:

$$
Y=1 / Z=G+j \omega C=G+j(2 \pi f) C
$$

where $Y$ is the admittance, $Z$ is the impedance, $G$ is the conductance, $\omega$ is the angular frequency, $f$ is the frequency and $C$ is the capacitance. Figure 2 shows the conductance characteristic as a function of frequency ( $G$ f) with various bias voltages $(0.2-5.0 \mathrm{~V})$ of the ITO/PTAA/Al Schottky diode. The $G-f$ characteristics indicated that conductance has remained almost constant up to a certain value of the low frequency. As the frequency was increased, the conductance of the PTAAbased diode showed a continuous increase over the applied bias voltages, hence the conductance is frequency dependent characteristics. The behavior of the $G-f$ characteristics is related to the long-range ordering which involving a translational motion with a sudden hopping [16]. The origin of the conductance stayed in the relaxation phenomena and when a mobile charge carrier hopped from origin to a new position, the charge remained in the state of displacement between two molecules, which then forming the hopping conductivity [30].

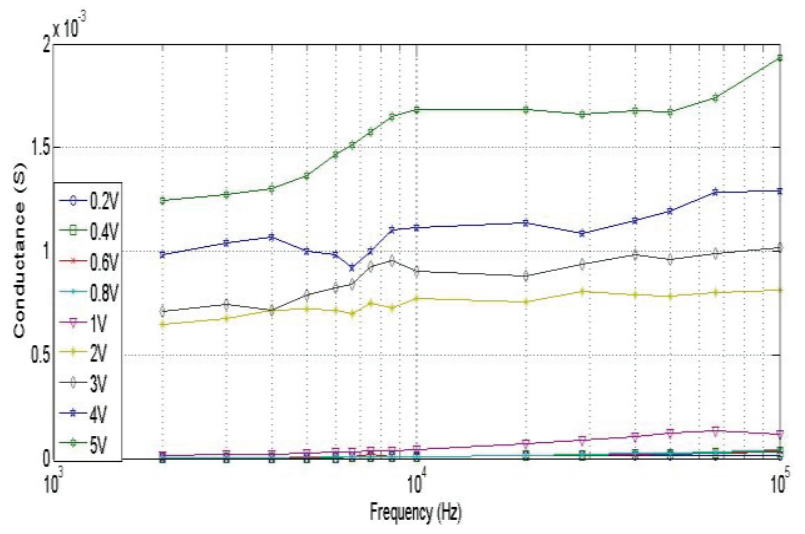

Fig. 2. Conductance characteristic as a function of frequency $(G-f)$ with various bias voltages $(0.2-5.0 \mathrm{~V})$ of the ITO/PTAA/Al Schottky diode.

Figure 3 shows the capacitance characteristics as a function of frequency $(C-f)$ of ITO/PTAA/Al Schottky diode in the bias voltage range from 0.2 to $5.0 \mathrm{~V}$. The $C$ $f$ characteristics indicated a decrease of the capacitance values as the frequency was increased $(<100 \mathrm{~Hz})$ and it remained constant at the high frequency range $(>10$ $\mathrm{kHz}$ ). The capacitance is defined by the quality of trap charge in the band of a device [31]. The high values of capacitance at low frequencies $(<100 \mathrm{~Hz})$ were attributed to the excess capacitance from the contribution of the interface trap charges, which depends on the frequency and the relaxation time of charge at interface state or the interface trap [32]. However, a continuous contribution of interface trap charges was limited by the high frequency region $(>10 \mathrm{kHz})$. As the small portion of interface charges, known as space charge capacitance, 
cannot follow the signal and negligibly small, only the interface state capacitance is contributing to the total capacitance and yield from the geometrical capacitance value [33].

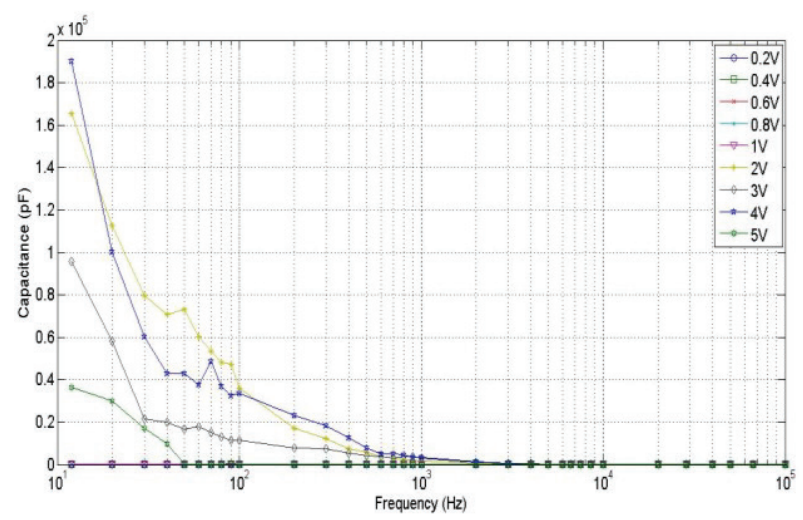

Fig. 3. Capacitance characteristics as a function of frequency $(C-f)$ of ITO/PTAA/Al Schottky diode in the bias voltage range from 0.2 to $5.0 \mathrm{~V}$.

\subsection{Voltage dependent conductance and capacitance}

Figure 4 and Figure 5 show the conductance $(G-V)$ and capacitance $(C-V)$ characteristics as a function of bias voltage at different frequencies for ITO/PTAA/Al Schottky diode, respectively. The $G-V$ characteristic indicated that the conductance values increase from 0.2 to $5.0 \mathrm{~V}$ at a frequency of $2 \mathrm{kHz}, 10 \mathrm{kHz}$ and $100 \mathrm{kHz}$, respectively as shown in Figure 4. Furthermore, the conductance value also increases with increasing frequency at a given bias voltage. On the other hand, the capacitance value of the diode within the range of $0.2 \mathrm{~V}$ and $5.0 \mathrm{~V}$ increases with decreasing frequency at a given voltage as shown in $C-V$ of Figure 5. At low frequency, capacitance exhibited a high value which indicated that the organic diode is turned on and the charges are trapped due to the threshold voltage at approximately 1.0 $\mathrm{V}$ and the charge had enough energy to pass through the depletion region at full turn-on voltage of $4.0 \mathrm{~V}$.

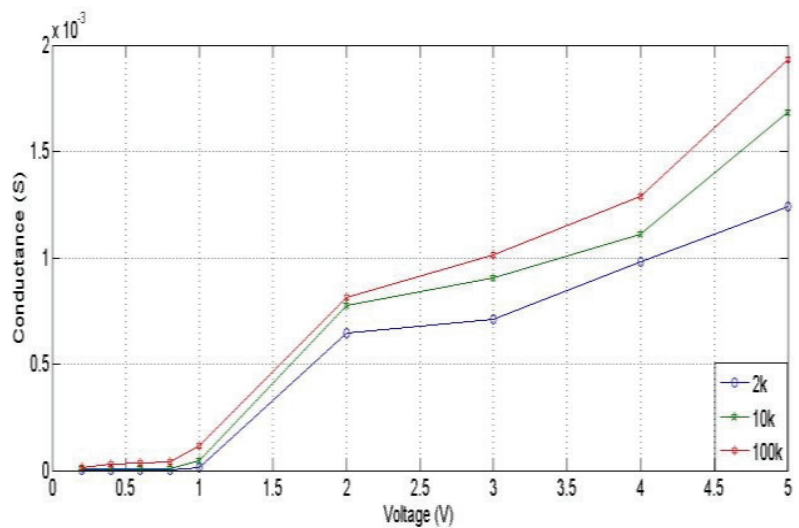

Fig. 4. Conductance characteristics as a function of bias voltage $(G-V)$ at different frequencies for ITO/PTAA/Al Schottky diode.

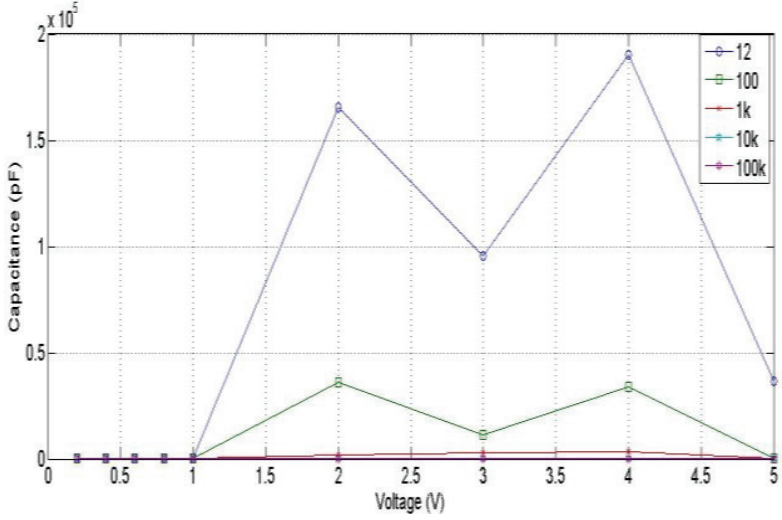

Fig. 5. Capacitance characteristics as a function of bias voltage $(C-V)$ at different frequencies for ITO/PTAA/Al Schottky diode.

This phenomenon is attributed to the existence of interface state traps, interfacial PTAA layer and series resistance effect of the Schottky diode [17]. In fact, that the charges at the interface states can follow the $\mathrm{AC}$ signal at low frequencies, the total excess capacitance is contributed by the interface charges in addition to the space charge capacitance. Thus, the capacitance is strongly dependent on the frequency at this low range. As the voltage is built-in in the diode, the capacitance achieves the highest value in the range between 2.0 to $4.0 \mathrm{~V}$ and this capacitance peak region is attributed to the imperfect ohmic contacts that add a contact resistance to the series resistance or only the interface states $[34,35]$. Furthermore, the values of the capacitance at the peak region decrease with increasing frequency. This is attributed to the interface states are limited by the high frequencies. In addition, both $G-V$ and $C-V$ characteristics show three regions which indicated the inversion, accumulation, and depletion regions. The capacitance and conductance values were changed at the accumulation and depletion regions, but remained constant in the inversion region.

\subsection{Frequency dependent series resistance and state density}

Comparing the real and imaginary part of the admittance of the parallel $R C$ circuit in Equation (1), the series resistance $\left(R_{S}\right)$ at strong accumulation region of the device is given by $[24,25]$ :

$$
R_{S}=G_{m} / G_{m}^{2}+\left(\omega C_{m}\right)^{2}
$$

where the value of $G_{m}$ and $C_{m}$ are the measured conductance and capacitance as a constant bias voltage at various frequencies. Figure 6 and Figure 7 show the series resistance as a function of frequency $\left(R_{S}-f\right)$ and bias voltage $\left(R_{S^{-}} V\right)$ for the ITO/PTAA/Al diode, respectively. These significant characteristics revealed the effects of the series resistance in the application of admittance-based measured methods. The $R_{S-} f$ characteristics indicated that the series resistance is depends on frequency and can be differentiated into two regions [36]. The value of series resistance decreases 
with the increasing frequency and remains almost constant in the higher frequency region for each bias voltage as shown in Figure 6. This attributed to the interfacial polymer layer and the interface states between polymer layer and contact interface, which cannot follow the ac signal and consequently cannot contribute to the diode. On the other hand, the values of series resistance are high at a low bias voltage ranging from 0.2 to $2.0 \mathrm{~V}$ due to intensive interface states at low frequency as shown in the $R_{S^{-}} V$ characteristics of Figure 7. However, series resistance almost disappears or negligibly small as the bias voltage increases further from 2.0 to $5.0 \mathrm{~V}$ which attributed to the trapped charges in interface state with low energy that unable to pass through the depletion region [33], thus this contributed to high values of capacitance in the voltage range.

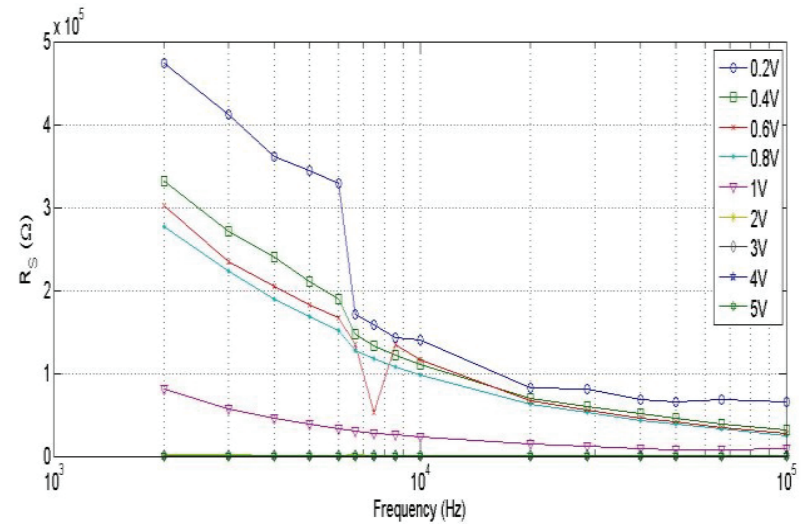

Fig. 6. Series resistance as a function of frequency $\left(R_{S}-f\right)$ for the ITO/PTAA/Al diode.

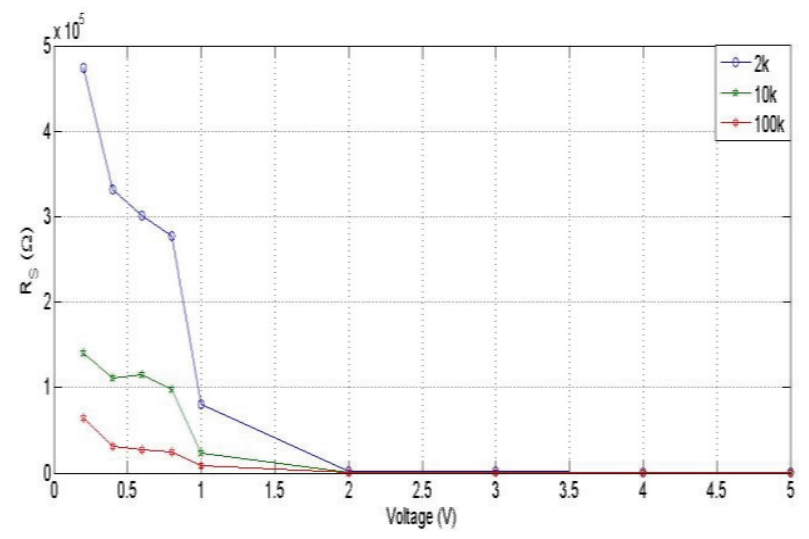

Fig. 7. Series resistance as a function of bias voltage $\left(R_{S^{-}} V\right)$ for the ITO/PTAA/Al diode.

In addition, frequency dependent of interface state density $\left(N_{S S}\right)$ was obtained using the Hill-Coleman method, where the interface states density values can be calculated by using the following equation [23]:

$$
N_{S S}=2 / g A \cdot G_{m, \max } /\left(G_{m, \max } C_{o x}\right)^{2}+\left(1-C_{m} / C\right)^{2}
$$

where, A is the area of the diode, $G_{m}$ and $C_{m}$ are the measured conductance and capacitance, respectively, and $C$ is the capacitance of polymer layer and can be obtained from $G-V$ and $C-V$ measurements in strong accumulation regions of a constant bias voltage of $4.0 \mathrm{~V}$ at constant frequency of $10 \mathrm{kHz}$ by using the following relation [18]:

$$
\left.C=C_{m a} \mid 1+G_{m a}^{2} /\left(\omega C_{m a}\right)^{2}\right\rfloor
$$

The interface states density as a function of frequency $\left(N_{S S}-f\right)$ is shown in Figure 8. The $N_{S S-} f$ characteristic indicated that the interface states density values almost constant at $2.8 \times 10^{12} \mathrm{eV}^{-1} \mathrm{~cm}^{-2}$ with slightly decrease with increasing frequencies. At low frequency, the interface states density depends on frequency, showing the high value of capacitance, which attributed to the excess capacitance resulting from the interface states. However, it is almost independent of frequency at high frequencies. Thus, the diode is frequency dependent interface states density, which similar behavior and comparable to that to the other organic Schottky diodes $[18,26,37]$, and currently further investigations to enhance the electrical responses of PTAA-based Schottky diode by adding the small molecule such as soluble pentacene to form a blend PTAA-pentacene system is currently undergoing [38].

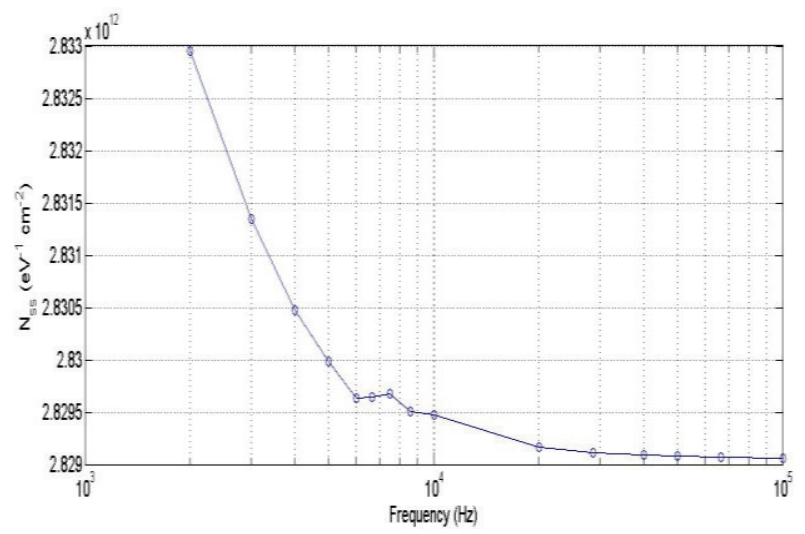

Fig. 8. Interface state density as a function of frequency $\left(N_{S S}-f\right)$ for the ITO/PTAA/Al diode.

\section{Conclusions}

In summary, we have successfully fabricated a metalorganic-metal (MOM) type Schottky diode based on poly(triarylamine) (PTAA) thin films by using the spin coating method. The frequency and voltage dependence conductance and capacitance characteristics of metalorganic-metal (ITO/PTAA/Al) Schottky diode were investigated in the frequency range of $12 \mathrm{~Hz}$ to $100 \mathrm{kHz}$ and bias voltage range of 0.2 to $5.0 \mathrm{~V}$, respectively at room temperature. The frequency dependent characteristics of the Schottky diode show that both conductance and capacitance were sensitive to frequency and voltage, especially at low frequency region, which attributed to the interface states at the polymer interface and series resistance. Thus, this behavior for both conductance and capacitance can follow an AC signal at low frequencies but cannot follow at high frequencies. Moreover, series resistance is dependent both frequency and bias voltage and have a significant effect on electrical characteristics of ITO/PTAA/Al MOM type Schottky diode. Moreover, the interface state density 
was found almost constant with slightly decrease with increasing frequency. This development of Schottky diode based on amorphous PTAA thin film has been realized and further improvements in the electrical responses should be possible by improving the thin film morphology and structure as well as the fabrication conditions for possible applications.

The authors would like to thank Artificial Intelligence Research Unit (AiRU) at Universiti Malaysia Sabah for the facilities provided.

\section{References}

1. X. Ye, H. Lin, X. Yu, S. Han, M. Shang, L. Zhang, Q. Jiang, J. Zhon, Synth. Met. 209, 337 (2015)

2. J. Takeya, T. Uemura, K. Sakai, Y. Okada, Thin Solid Films 554, 19 (2014)

3. M. U. Hassan, Y. C. Liu, K. U. Hasan, H. Butt, J. F. Chang, R. H. Friend, Nano Energy 21, 62 (2016)

4. P. S. K. Amegadze, Y. Y. Noh, Thin Solid Films 556, 414 (2014)

5. T. Ohe, M. Kuribayashi, R. Yasuda, A. Tsuboi, K. Nomoto, K. Satori, M. Itabashi, J. Kasahara, Appl. Phys. Lett. 93, 053303 (2008)

6. Y. Xu, T. Minari, K. Tsukagoshi, R. Gwoziecki, R. Coppard, F. Balestra, G. Ghibaudo, Org. Electron. 12, 2019 (2011)

7. C. Celle, C. Suspene, J. Simonato, S. Lenfant, M. Ternisien, D. Vuillaume, Org. Electron. 10, 119 (2009)

8. K. A. Mohamad, H. T. Hoh, B. K. Ghosh, A. Alias, I. Saad, J. Adv. Res. Appl. Mech. 13, 24 (2015)

9. E. M. Barea, G. Garcia-Belmonte, M. Sommer, S. Huttner, H. J. Bolink, M. Thelakkat, Thin Solid Films 518, 3315 (2010)

10. Y. H. Kim, J. E. Anthony, S. K. Park, Org. Electron. 13, 1152 (2012)

11. J. H. Kwon, S. I. Shin, J. Choi, M. H. Chung, T. Y. Oh, K. H. Kim, M. J. Cho, K. N. Kim, D. H. Choi, B. K. Ju, Org. Electron. 10, 729 (2009)

12. B. Arredondo, B. Romero, G. D. Pozo, M. Sessler, C. Veit, U. Wurfel, Sol. Energy Mater. Sol. Cells 128, 351 (2014)

13. M. Ullah, D. M. Taylor, R. Schwodiauer, H. Sitter, S. Bauer, N. S. Sariciftci, T. B. Singh, J. Appl. Phys. 106, 114505 (2009)

14. K. P. Chandra, K. Prasad, R. N. Gupta, Physica B 388, pp. 118 (2007)

15. J. H. Ahn, J. U. Lee, T. W. Kim, Curr. Appl. Phys. 7, 509 (2007)

16. H. Dogan, N. Yildirim, I. Orak, S. Elagoz, A. Turut, Physica B 457, 48 (2015)

17. B. Baris, Physica B 426, 132 (2013)

18. S. Zeyrek, E. Acaroglu, A. Altindal, S. Birdogan, M. M. Bulbul, Curr. Appl. Phys. 13, 1125 (2013)
19. T. Tunc, S. Altindal, I. Uslu, I. Dokme, H. Uslu, Mater. Sci. Semicond. Process. 14, 139 (2011)

20. T. Kilicoglu, M. E. Aydin, Y. S. Ocak, Physica B 388, 244 (2007)

21. K. K. Hung, Y. C. Cheng, J. Appl. Phys. 62, 4204 (1987)

22. S. Kar, S. Varma, J. Appl. Phys. 58, 4256 (1985)

23. W. A. Hill, C. C. Coleman, Solid State Electron. 23, 987 (1980)

24. E. H. Nicollian, A. Goettzberger, Bell Sys. Tech. J. 46, 1055 (1967)

25. E. H. Nicollian, A. Goettzberger, Appl. Phys. Lett. 7, $216(1965)$

26. M. S. P. Reddy, J. H. Lee, J. S. Jang, Synth. Met. 185-186, 167 (2013)

27. W. G. Osiris, A. A. M. Farag, I. S. Yahia, Synth. Met. 161, 1079 (2011)

28. H. Xio, S. Huang, Mater. Sci. Semicond. Process. 13, 395 (2010)

29. S. Sonmezoglu, S. Senkul, R. Tas, G, Cankaya, and M. Can, Thin Solid Films 518, 3475 (2010)

30. A. Cherif, S. Jomni, H. Saghrouni, W. Belgacem, K.Khirouni, L. Beji, J. Alloys Compd. 685, 28 (2016)

31. Y. S. Lee, J. H. Park, S. C. Jong, J. Korean Phys. Soc. 42, 294 (2003)

32. C. Bilkan, A. Gumus, S. Altindal, Mater. Sci. Semicond. Process. 39, 484 (2015)

33. S. Karatas, A. Turut, Microelectron. Reliab. 50, 351 (2010)

34. E. J. Miller, X. Z. Dang, H. H. Wieder, P. M. Asbeck, E. T. Yu, G. J. Sullivan, J. M. Redwing, J. Appl. Phys. 87(11), 8070 (2000)

35. X. Wuand, E. S. Yang, J. Appl. Phys. 65, 3560 (1989)

36. V. S. Reddy, S. Das, S. K. Ray, A. Dhar, J. Phys. D Appl. Phys. 40, 7687 (2007)

37. C. Tozlu and A. Mutlu, Synth. Met. 211, 99 (2016)

38. K. A. Mohamad, H. T. Hoh, A. Alias, B. K Ghosh, I. Saad, H. Fukuda, Ext. Abstr. Solid State Devices and Materials, 313 (2016) 